\section{LA VERRUGA PERUANA: INVESTIGACIÓN HISTOPATOLÓGICA REALIZADA POR CHILE EN EL SIGLO XIX}

\author{
THE VERRUGA PERUANA: \\ HISTOPATOLOGICAL RESEARCH MADE BY \\ CHILE IN THE NINETEENTH CENTURY
}

\section{David Salinas Flores ${ }^{1, a}$}

Sr. Editor. Recientemente se ha publicado una revisión sobre la investigación sobre la verruga peruana realizada antes de Carrión, la cual se enfocó principalmente al campo de la histopatología, sin embargo, tuvo una omisión histórica importante: la investigación histopatológica realizada en Chile a fines del siglo XIX ${ }^{(1)}$.

La verruga peruana es una infección que tiene una localización geográfica determinada por la distribución del posible vector, el mosquito Lutzomya verrucarrum, de ahí que solo se reporte en Perú, Colombia y Ecuador, por ello, no hay mayores estudios chilenos en la actualidad sobre esta enfermedad. Sin embargo, a fines del siglo XIX, científicos chilenos desarrollaron una importante investigación pionera sobre la verruga peruana, debido que en esa época, esta infección era un problema de salud pública para Chile, debido a la migración de ciudadanos chilenos a zonas endémicas de verruga peruana como consecuencia de la construcción del ferrocarril trasandino en Perú y la guerra con Chile ${ }^{(2)}$.

Los científicos chilenos que investigaron la verruga peruana fueron el Dr. Vicente Izquierdo, quien se enfocó en los aspectos histopatológicos y microbiológicos, y Luis Sanfurgo Reyes, al momento, un estudiante de medicina, quien asumió la parte clínica y la terapéutica. El Dr. Vicente Izquierdo, formado en la Escuela de Medicina de la Universidad de Chile, fue becado para estudiar en Alemania, donde se doctoró en 1879. Su retorno a Chile coincidió con la guerra con Perú por lo que ingresó al servicio sanitario del ejército donde conoció el problema de la verruga peruana que afectaba a soldados chilenos ${ }^{(3)}$.

El estudiante de medicina Luis Sanfurgo, en su tesis de bachiller de medicina sobre la verruga peruana, describe que entregó muestras de verruga peruana, al Dr. Vicente Izquierdo para que las investigara: Recojimos con el mayor esmero una cantidad de muestras de las diferentes formas... para confiarlos al profesor don Vicente Izquierdo quien ha hecho un prolijo estudio...y encontrado, por fin, la causa especial de esta enfermedad. Figura 1.

\footnotetext{
Universidad Nacional Mayor de San Marcos, Lima, Perú.

a Médico cardiólogo

Recibido: 13-07-14 Aprobado: 23-07-14
}

Citar como: Salinas Flores D. La verruga peruana: la investigación histopatológica realizada por Chile en el siglo XIX. Rev Peru Med Exp Salud Publica. 2014;31(3):609-10.

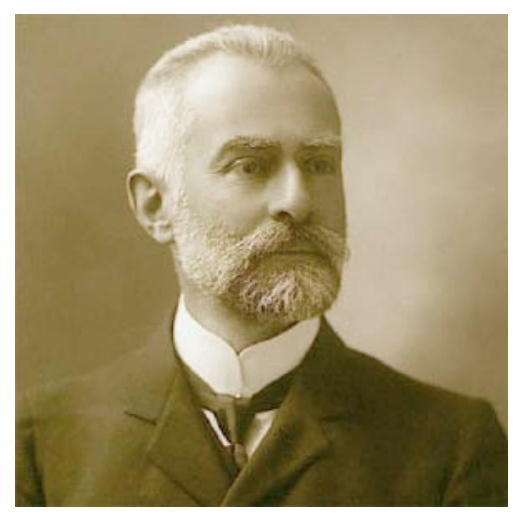

Figura 1. Dr. Vicente Izquierdo. Fuente: Museo Nacional de Medicina Enrique Laval (Internet) Santiago, Chile. 2014.

Izquierdo inicia así, una investigación sobre esta enfermedad, la cual fue publicada con el nombre de Histopatología de la verruga peruana en la prestigiosa revista alemana los Archivos de Virchow y, luego, expuesta en el primer congreso médico chileno, él describe: Los tumores verrucosos se desarrollan a expensas del tejido conjuntivo epidérmico ó sub - dérmico, con una gran vascularización y semejando su constitución a la del sarcoma (sic) ${ }^{(4)}$.

Es importante recordar esta investigación histopatológica por tres razones: primero, fue una de las primeras aproximaciones al estudio de esta enfermedad desde el punto de vista histopatológico; en segundo lugar, describe acertadamente las semejanzas de la verruga peruana con los sarcomas, lo que ha sido confirmado recientemente, y finalmente, porque tuvo un papel crucial en la historia de la investigación de la verruga peruana, dado que por ser chilena, estimuló a Daniel A. Carrión a realizar su histórico experimento sobre esta enfermedad ligada a Perú ${ }^{(4,5)}$.

\section{REFERENCIAS BIBLIOGRÁFICAS}

1. Takano J. Bartonelosis humana: antes y después de Daniel Alcides Carrion. Rev Per Med Exp Salud Publica. 2014;31(2):385-9.

2. Salinas D. Daniel Alcides Carrion en las publicaciones biomédicas chilenas: errores históricos. Rev Med Chile. 2014;142(5):657-8.

3. Museo Nacional de Medicina Dr. Enrique Laval. Dr. Izquierdo, Vicente (1850-1926) [Internet]. Santiago; 2014 [citado el 11 de julio de 2014]. Disponible en: http://www.museomedicina. cl/home/index.php/historia-de-la-medicina/181-dr-vicenteizquierdo-1850-1926.html

4. Izquierdo V. Spaltpilze bei der Verruga peruana. Virchow's Arch. 1885;99:411-8.

5. Arias-Stella J, Lieberman PH, Garcia-Caceres U, Erlandson RA, Kruger H, Arias-Stella J Jr. Verruga peruana mimicking malignant neoplasms. Am J Dermatopathol. 1987;9(4):279-91.

Correspondencia: David Salinas Flores

Dirección: Calle Federico Villarreal \# 592 Urb. Ingenieria, San Martín de Porres, Lima 31, Perú.

Teléfono: +511996371790

Correo electrónico:dsalinas2009@yahoo.com 